\title{
Impulsive Breakdown in Water: Optimisation of Energy Delivery for High Acoustic Output
}

\author{
I. V. Timoshkin, Y. Sun, M. J. Given, M. P. Wilson, \\ T.Wang, S. J. MacGregor \\ Electronic and Electrical Engineering Department \\ University of Strathclyde, Glasgow, UK \\ igor.timoshkin@strath.ac.uk
}

\author{
N. Bonifaci \\ G2E Laboratory, CNRS \\ Grenoble, France \\ nelly.bonifaci@g2elab.grenoble-inp.fr
}

\begin{abstract}
The high voltage impulsive breakdown process in water is complex, with the nature of the impulsive breakdown depending upon the magnitude, polarity and rise time of the HV impulses, the water conductivity, and the electrode topology. In the case of $\mu \mathrm{s}$ and sub- $\mu \mathrm{s}$ high voltage impulses of sufficient magnitude, the breakdown develops through the formation of plasma streamers in the water. When the first streamer crosses the entire inter-electrode gap, the energy released in the breakdown channel transforms this channel into a gas/vapor cavity, which pulsates and radiates acoustic impulse(s). Optimisation of the hydrodynamic (period of cavity oscillation) and acoustic (peak magnitude of the acoustic impulse(s)) parameters is required for practical applications of these underwater spark discharges. The present paper analyses the functional behavior of the period of cavity oscillation and the peak magnitude of the acoustic impulse for spark discharges generated by self-triggered underwater discharges (free discharges), spark discharges triggered by air bubbles injected into the inter-electrode gap, and wire-guided discharges. The advantages and limitations of these methods of generation of underwater acoustic impulses by spark discharges are discussed.
\end{abstract}

Keywords-Breakdown in water, air bubbles, wire-guided discharge, acoustic efficiency

\section{INTRODUCTION}

Impulsive breakdown of water has been intensively studied for several decades. Typically, two main phases are distinguished in the breakdown process: the pre-breakdown phase, which is completed by the formation of the conductive breakdown channel between the electrodes immersed in water; and the post-breakdown phase, during which the energy is released into the breakdown channel, resulting in the growth of a gaseous cavity and the generation of strong acoustic impulse(s). In the case of selfbreakdown (free) underwater discharges and air-bubble stimulated discharges, the first phase is typically associated with the development and propagation of plasma filaments (streamers) across the inter-electrode gap. In the case of wire-guided discharges, the plasma channel between the electrodes is developed by the rapid heating and disintegration of the conductive wire initially placed between the HV and ground electrodes. In all cases, the second, post-breakdown phase is characterised by the deposition of energy into the established conductive plasma channel, causing rapid heating and expansion of this channel.
The expanding plasma channel generated through selfbreakdown, gas-bubble-triggered or wire-guided breakdowns drives a strong acoustic pressure impulse into the surrounding water. In the 1960s, several research papers were focused on the development of different practical applications of such impulses. For example, in [1 - 3], the electro-hydraulic pressure impulses generated by underwater free spark discharges were used for comminution of different solid materials (rocks and minerals). This discharge method of crushing and breakage of solids was developed further by several research groups: spark plasma discharges were used for recycling of solid materials [4-6] and for electric spark peening, [7]. In addition, underwater pressure impulses can be used for medical and biological applications such as lithotripsy, inactivation of microorganisms [8], and for environmental applications including water treatment [9]. In the field of pulsed power, operation of water-filled crowbar switches is based on impulsive breakdown of water [10].

The present paper provides an analysis of different approaches that could be used to minimise the energy losses, caused by conduction between the electrodes, before formation of the breakdown channel. The post-breakdown characteristics of the transient plasma channel, i.e. the plasma resistance, the period of the gas cavity oscillations, and their functional dependencies on the pulsed power circuit parameters, are also presented and discussed. For all types of discharges considered in this paper, the functional dependencies of the period of cavity oscillations, the resistance of the plasma channel, and the peak acoustic magnitude, on the energy deposited into the spark channel have been obtained. The paper provides a comprehensive picture of the functional behavior of the hydrodynamic and acoustic parameters for the three discharge types considered. The results obtained will be helpful in optimising and tailoring the parameters of pulsed power circuits and underwater discharges for different practical and industrial applications.

\section{BREAKDOWN WAVE-FORMS}

In the present work, the hydrodynamic and acoustic parameters of the underwater spark discharges generated by 
free breakdown of self-triggered gaps, spark discharges stimulated with injected air bubbles, and wire-guided discharges are considered. The comparative analysis of these discharges is based on experimental results obtained using a capacitive energy storage pulsed power supply. Three different storage capacitances $(155 \mathrm{nF}, 266 \mathrm{nF}$, and $533 \mathrm{nF})$ and three different charging voltages $(25 \mathrm{kV}$, $30 \mathrm{kV}$, and $35 \mathrm{kV}$ ) were used. This wide range of energies available to the discharges has been tested using all three types of discharge (free discharges, air-bubble-stimulated discharges, and wire-guided discharges).

The experimental set-up including the pulsed power system and the electrical and acoustic diagnostic equipment has been described in detail in [13]. The electrode systems used are described: for free discharges in [13]; for airbubble discharges in [14]; and for wire-guided discharges in [15]. Examples of the current and voltage waveforms for all types of discharge are shown in Fig. 1. It can be seen that, in the case of the same inter-electrode distance, $15 \mathrm{~mm}$ (for free discharges and air-bubble triggered discharges) and $15 \mathrm{~mm}$ length of the guiding wire, the pre-breakdown time is significantly shorter for the air-bubble triggered discharges than for the free discharges. The time of the wire disintegration in the case of wire-guided discharges is significantly shorter than the pre-breakdown time for both the free and air-bubble stimulated discharges. Thus, the prebreakdown energy losses, which occur due to conduction across the gap in the interval between voltage application and breakdown, can be substantially reduced by using airbubble stimulated and wire-guided discharges.

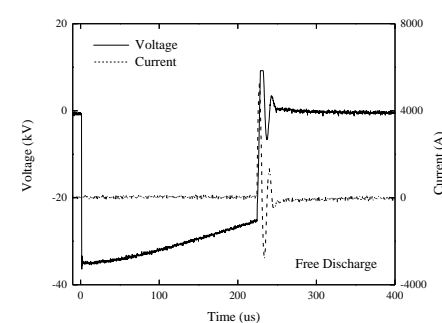

(a)

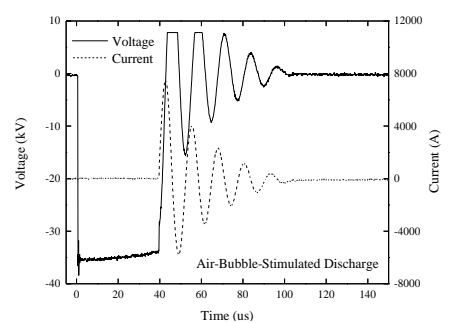

(b)

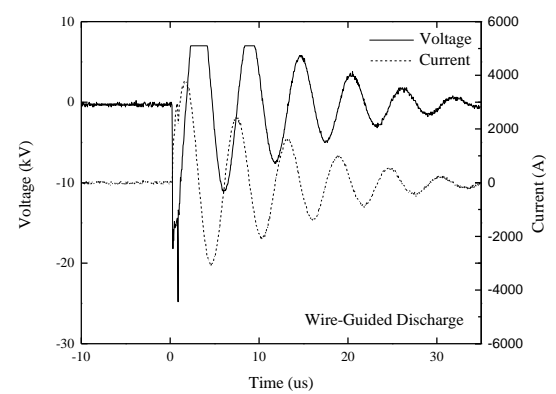

(c)

Fig. 1. Voltage and current waveforms for (a) free-discharge with $15 \mathrm{~mm}$ gap, $25 \mathrm{kV}$ breakdown voltage, $566 \mathrm{nF}$; (b) air-bubble-stimulated discharge with $15 \mathrm{~mm}$ gap, $34 \mathrm{kV}$ breakdown voltage, $566 \mathrm{nF}$; (c) wire-guided discharge with $15 \mathrm{~mm}$ gap, $23 \mathrm{kV}$ breakdown voltage, $155 \mathrm{nF}$.
The pre-breakdown phase of the spark discharge is completed by the formation of the conductive plasma channel between the electrodes, which results in the appearance of a high conduction current in this channel, leading to its expansion and transformation into a gas cavity. The dynamics of the plasma/gas cavity formed by free discharges in water have been intensively studied. In [11] and [13], it was shown that the transient process of the energy deposition into the plasma channel can be described using a constant resistance approximation, i.e. assuming that the resistance of the plasma is constant during the current oscillations. This approach simplifies the hydrodynamic description of the plasma/gas cavity, allowing for analysis of the period of cavity oscillation and the cavity radius. The cavity radiates the primary acoustic impulse during its expansion phase, and a secondary impulse during its collapse phase. Typically, several expansion-collapse cycles of the gas cavity are observed.

For different practical applications, it is important to predict and optimise both the hydrodynamic and the acoustic parameters of the pulsating plasma/gas cavity developed by the spark discharge. Fig. 2 summarises the results of measurements of the period of cavity oscillation for all 3 types of discharges: free discharge (FD), airbubble-stimulated discharge (ABSD), and wire-guided discharge (WGD). Fig. 2 shows the period of cavity oscillation as a function of the constant plasma channel resistance, $R_{p l}$, derived for each discharge. The methodology for obtaining the plasma resistance is described in [13].

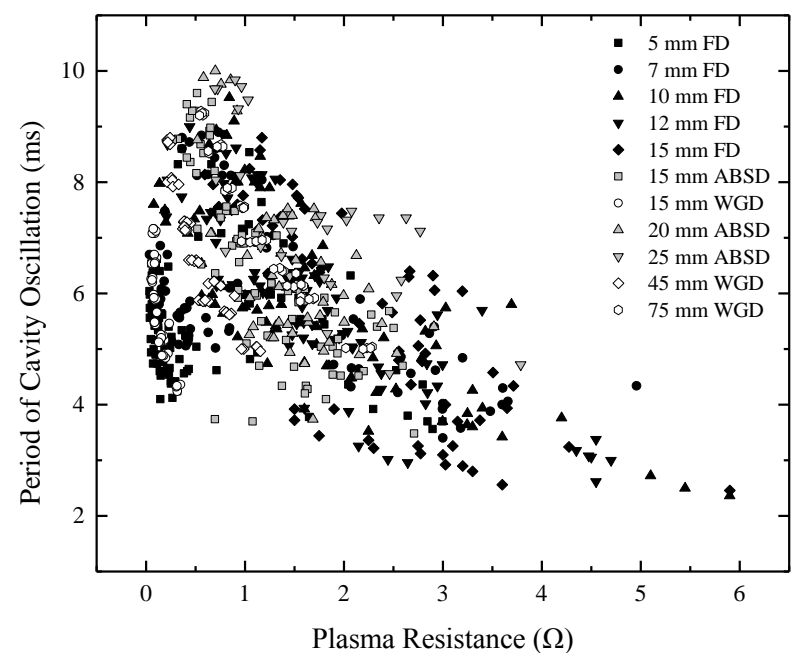

Fig.2. The relationship between the period of cavity oscillation and the plasma resistance for FD, ABSD and WGD.

Fig. 2 shows that the period of cavity oscillation is a non-linear function of the plasma resistance, which initially increases, then decreases as $R_{p l}$ increases. It was found in [13] that the maximum values of the period of cavity oscillations for free discharges in water are achieved at plasma resistances which are almost twice the value of the resistance of the pulsed power driving circuit. Further increase in the plasma resistance results in the reduction of 
the period of cavity oscillation, due to lower values of the energy available at the discharge for these "tail" values of $R_{p l}$. This non-linear behavior of the period of cavity oscillation is dictated by a combination of several factors, including the energy transfer efficiency from the pulsed power system to the plasma channel, and the breakdown voltage, which defines the energy available to the spark discharge. The observed non-linear behavior has been discussed for free discharges in [13].

Fig. 3 shows the peak magnitude of the primary acoustic signal generated by the expanding plasma cavity. This signal was measured using an un-calibrated piezo-electric transducer (Pinducer VP1093), which generates an output voltage signal which is proportional to the pressure in the underwater acoustic signal. Detailed descriptions of the acoustic measurements are provided in [13], [14] and [15]. The peak magnitude of the voltage signal (which is proportional to the acoustic signal) as a function of the derived plasma resistance is shown in Fig. 3 for all 3 types of discharges. The black points represent the peak acoustic magnitudes generated by free discharges in water, the grey points represent the peak acoustic magnitudes generated by air-bubble-triggered discharges, and the open points represent the peak acoustic magnitudes generated by wireguided discharges. It can be seen that the "optimal" plasma resistance values are again approximately twice the value of the resistance of the pulsed power circuit. The wire guided discharges produce the strongest acoustic signals. The peak magnitudes of the acoustic impulses produced by airbubble-stimulated discharges are typically higher than those for free discharges in water.

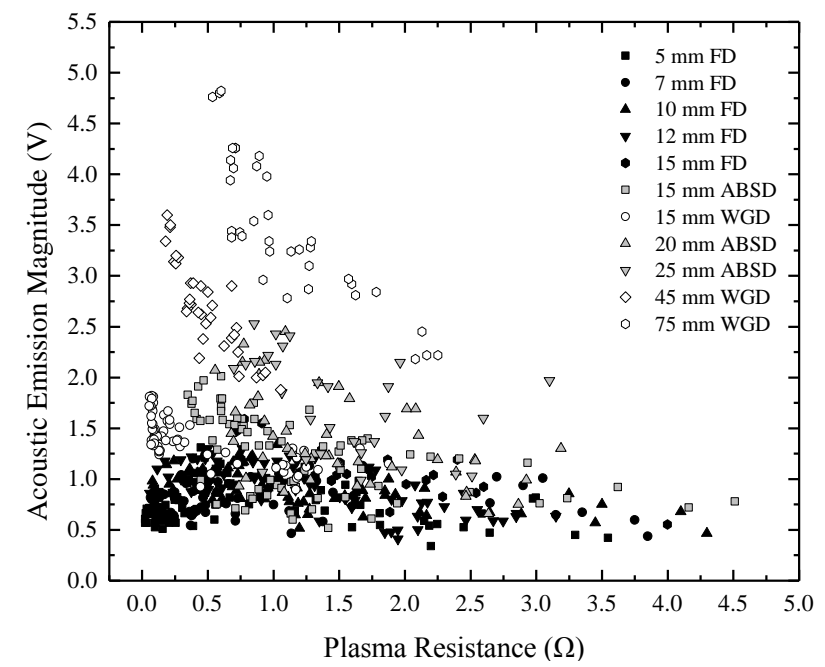

Fig. 3. Acoustic emission magnitude as a function of plasma resistance for FD, ABSD and WGD.

The main reason for the stronger acoustic output produced by the wire-guided discharges was that breakdown could be achieved with longer inter-electrode distances by using conductive wires placed between the electrodes. As can be seen from Fig. 3, in the case of the wire-guided discharges, a $75 \mathrm{~mm}$ inter-electrode gap was broken down using the same charging voltage and energy storage capacitance as used for free discharges in a $15 \mathrm{~mm}$ gap, the maximum gap length for consistent free discharge breakdown. This longer inter-electrode distances result in a larger surface area of the expanding plasma channel and, therefore, a larger acoustic radiation area. In addition, due to the rapid breakdown and, thus, higher breakdown voltages, more energy is available for deposition into the plasma channel. Therefore, the wire-guided discharges provide, as expected, the strongest acoustic signals. Air-bubblestimulated discharges also allow longer inter-electrode distances to be used as compared with free discharges, thus resulting in stronger acoustic signals than those for free discharges.

Using the current wave-forms and the constant resistance of the plasma channel, the energy deposited into the spark discharge can be calculated [13]. This energy has been obtained for all the breakdown events recorded for the three types of discharge, allowing the functional behavior of the period of cavity oscillation and the peak acoustic magnitude to be obtained. Fig. 4 shows the period of cavity oscillations for free, air-bubble-stimulated and wire-guided discharges, plotted as a function of the energy transferred to the discharge. As before, the darker (black) symbols represent free discharges, the lighter (grey) symbols represent air-bubble-stimulated discharges, and the open symbols represent wire-guided discharges. As Fig. 4 shows, there is a generic scaling, $T_{c a v}(W)$, for all types of discharge, i.e. the functional behavior, $T_{c a v}(W)$, for all three types of underwater discharge can be described by a single analytical function [13].

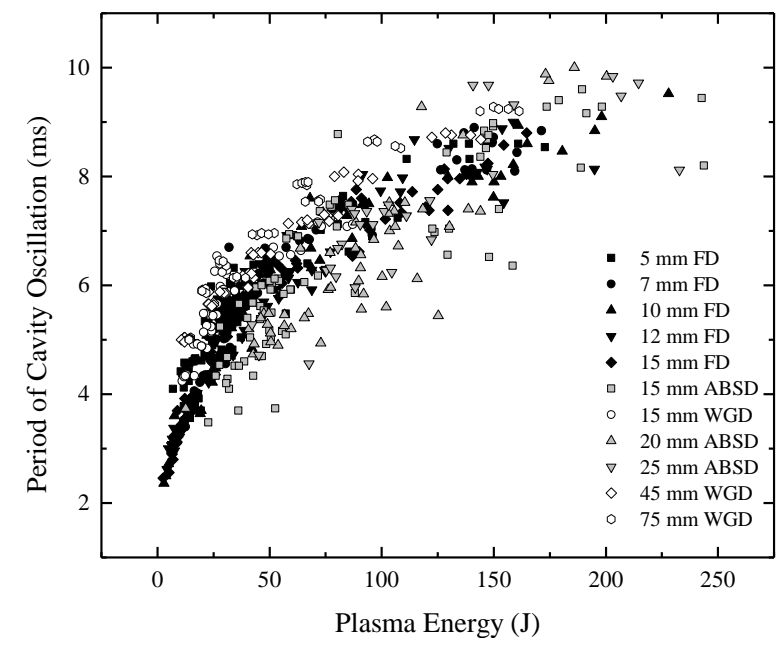

Fig. 4. Relationship between period of cavity oscillation and plasma energy for FD, ABSD and WGD.

However, the situation is different for the peak acoustic magnitude. This peak acoustic signal is shown in Fig. 5 as a function of the energy available in the discharge. Free discharges generate acoustic signals with the lowest magnitude as compared with the air-bubble-stimulated and 
wire-guided discharges (for the same values of energy transferred to the discharge cavity). The wire-guided discharges produce the strongest acoustic signal amongst these three types of discharge (again, for identical energy transferred to the discharge channel). Thus, due to the lower pre-breakdown losses and the higher acoustic output achieved for a given energy input in longer inter-electrode gaps, the wire-guided discharges provide the most efficient conversion of the energy released in the plasma channel into acoustic energy in the radiated acoustic impulse.

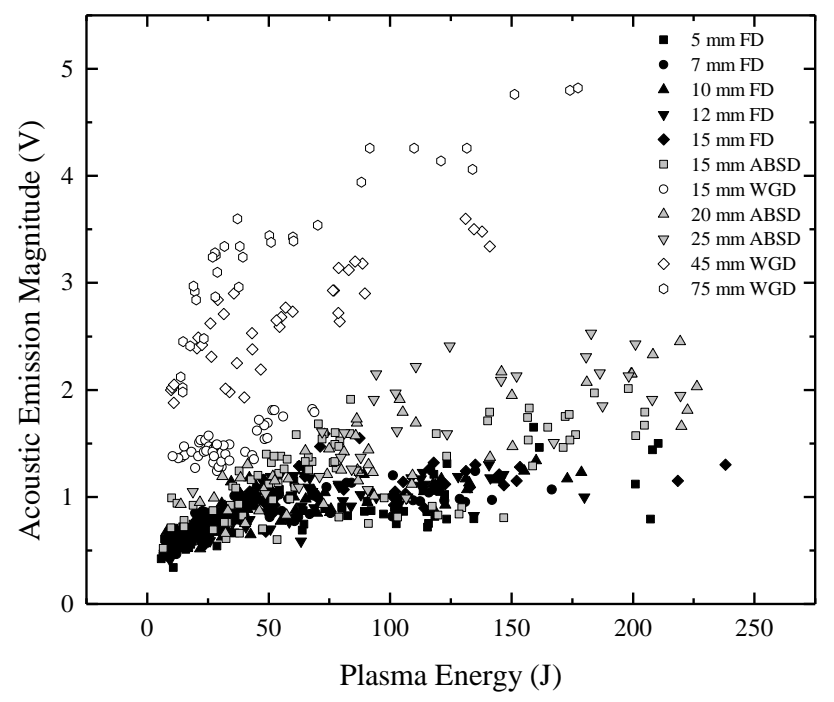

Fig.5. Acoustic emission magnitude as a function of plasma energy for FD, ABSD and WGD.

\section{CONCLUSIONS}

This paper presents a cumulative analysis of the hydrodynamic and acoustic characteristics of free, airbubble-stimulated and wire-guided discharges in water. The period of cavity oscillation and the peak acoustic pressure have been presented as functions of the plasma resistance and the energy available in the discharge. It is shown that due to larger radiating surface area (longer inter-electrode distances) achieved in the case of air-bubble-stimulated discharges and for wire-guided discharges in particular, the efficacy of conversion of the energy delivered into the plasma channel into the acoustic energy that the channel radiates is increased. Wire-guided discharges, therefore, have the advantage of providing the highest peak acoustic magnitude for a given pulsed power supply, as compared with two other types of discharge. However, wire-guided discharges require a new wire to be placed between the electrodes for each shot, which may reduce the practical applicability of this method. If repetitive discharges are required, the air-bubble-stimulated method may provide the optimal solution, as using this approach, discharges of increased length and acoustic output compared to free discharges can be generated at the same repetition rate as free discharges.

\section{REFERENCES}

[1] B. H. Bergstrom, "The electrohydraulic crusher", Eng. and Mining J., vol.162, pp.134-140, 1961.

[2] W. Carley-Macauly, J. W. Hitchon, N. G. Maroudas, "Energy consumption in electrohydraulic crushing", Trans. of Inst. of Chem. Eng., vol.44, pp.T395-T404, 1966.

[3] K. W. Carley-Macauly, "Electrohydraulic crushing", Chem. and Proc. Eng., vol.48, pp.87-92, 1968.

[4] H. Bluhm, W. Fre , H. Giese, P. Hoppe, C. Schultheis, R. Strassner, "Application of pulsed HV discharges to material fragmentation and recycling”, IEEE Trans. Dielectr. Electr. Insul., vol.7, no.5, pp. 625$636,2000$.

[5] I. Lisitsyn, T. Muraki, H. Akiyama, "Wire induced flashover as a source of shock waves for destruction of solid materials," Jpn. J. Appl. Phys., vol.36, pp.1258-1263, 1997.

[6] M. Wilson, L. Balmer, M. Given, S. MacGregor, I. Timoshkin, “An investigation of spark discharge parameters for material processing with high power ultrasound", Minerals Engineering, vol.20, n.12, pp. 1159-1169, 2007.

[7] I. V. Timoshkin, S. J. Macgregor, "Apparatus and method for electric spark peening of gas turbine components", Patent No. US 8,257,050 B2, 2012.

[8] S. Gillard, M. Speck, "Inactivation of microorganisms by electrohydraulic shock", Appl. Microbiology, vol.15, no.5, pp.1031$1037,1967$.

[9] T. Izdebski, M. Dors, J. Mizeraczyk, "River water remediation using electrohydraulic discharges or ozonation", IEEE Trans. On Plasma Sci., vol.39, no.3, pp.953-959, 2011

[10] S. Turnbull, S. MacGregor, F. Tuema, A. PcPhee, "A self-activated high-voltage high energy crow-bar", J. Phys. D: Appl. Phys., vol. 33, pp.1410-1416, 2000.

[11] I. V. Timoshkin, R. A. Fouracre, M. J. Given, S. J. MacGregor, "Hydrodynamic modelling of transient cavities in fluids generated by high voltage spark discharges", J. Phys. D: Appl. Phys., vol. 39, pp. 4808-4817, 2006.

[12] I. V. Timoshkin, S. J. MacGregor, M. J. Given and R. A. Fouracre, "High Power Ultrasound Impulses Induced by Wire-Guided Spark Discharges in Water," 2007 IEEE 34th International Conference on Plasma Science (ICOPS), Albuquerque, NM, 2007, pp. 145-145.

[13] Y. Sun, I. Timoshkin, M. Given, M.P. Wilson, T. Wang, S. J. MacGregor, N. Bonifaci, "Impulsive discharges in water: acoustic and hydrodynamic parameters", IEEE Transactions on Plasma Science, vol. 44, no. 10, pp. 2156-2166, 2016

[14] Y. Sun, I. Timoshkin, M. Given, M.P. Wilson, T. Wang, S. J. MacGregor, N. Bonifaci, "Hydrodynamic Parameters of AirBubble Stimulated Underwater Spark Discharges", IEEE Int. Conf. on Dielectric Liquids, Manchester, UK, 2017

[15] Y. Sun, I. Timoshkin, M. Given, M.P. Wilson, T Wang, S. J. MacGregor, N. Bonifaci, "Wire-Guided Discharges in Water for Generation of Acoustic Impulses", Submitted in Trans. on Plasma Sci., 2017. 